\title{
Experimental and Numerical Analysis of Hole Emission Process from Carbon-related Traps in GaN Buffer Layers
}

\author{
A. Chini, G. Meneghesso, Fellow, IEEE, M. Meneghini, Senior Member, IEEE, F. Fantini, Senior \\ Member, IEEE, G. Verzellesi, Senior Member, IEEE, A. Patti, F. Iucolano
}

\begin{abstract}
The role of carbon related traps in GaN-based ungated HEMT structures has been investigated both experimentally and by means of numerical simulations. A clear quantitative correlation between experimental data and numerical simulations has been obtained. The observed current decrease in the tested structure during backgating measurements has been explained simply by means of a thermally activated hole-emission process with $E_{A}=0.9 \mathrm{eV}$, corresponding to the distance of the acceptor-like hole-trap level from the $\mathrm{GaN}$ valence band. Moreover, it has been demonstrated by means of electrical measurements and numerical simulations that only a low percentage of the nominal Carbon doping levels induces the observed current reduction when negative substrate bias are applied to the tested structure.
\end{abstract}

Index Terms-Wide band gap semiconductors, numerical simulation, carbon doping, trapping phenomena

\section{INTRODUCTION}

G AN-BASED high electron mobility transistors (HEMTs)are of increasing interest for the development of low on-resistance and low-switching losses devices for power switching applications $[1,2]$. One of the mandatory steps in order to realize an excellent solid-state power switch is the formation of a semi-insulating buffer layer which has to be able to both reduce the device leakage currents in off-state conditions as well as to withstand the large device operating voltages. Carbon doping, which induces a complex set of

This work was partially supported by the ENIAC JOINT UNDERTAKING project: E2COGaN - Energy Efficient Converters using GaN Power Devices.

A. Chini and F. Fantini are with the "Enzo Ferrari" Engineering Department of the University of Modena and Reggio Emilia, Via Vivarelli 10 41125 Modena, Italy (e-mail: alessandro.chini@unimore.it; fausto.fantini@unimore.it).

G. Meneghesso and M. Meneghini are with the Department of Information Engineering of the University of Padova, Via Gradenigo 6/B, 35131 Padova, Italy (e-mail: gauss@dei.unipd.it; menego@dei.unipd.it).

G. Verzellesi is with the Department of Sciences and Methods for Engineering of the University of Modena and Reggio Emilia, Via Amendola 2

Pad. Morselli, 42122 Reggio Emilia, Italy (e-mail: giovanni.verzellesi@unimore.it).

A. Patti and F. Iucolano are with STMicroelectronics, Stradale Primosole 50, $95121 \quad$ Catania, Italy (e-mail: alfonsto.patti@st.com; ferdinando.iucolano@st.com).

A. Chini, G. Meneghesso, M. Meneghini, F. Fantini and G. Verzellesi are also with Consorzio Interuniversitario per la Nanoelettronica - IUNET, Italy. acceptor trap levels located in the lower-half of the GaN bandgap, has been proposed [3] and it is widely used [4-6] in order to fabricate high insulating buffer layers. Nevertheless, its introduction has also been linked to device dynamic on resistance increase $[4,5]$ which represent a strong limitation in order to fully exploit the GaN-based devices capability for efficient power switching operation.

In the last years, several papers have been dealing with the understanding and modeling of trapping phenomena which are likely to be related to Carbon doping. Numerical simulations presented in [7] showed that a C-doped GaN buffer can cause a significant current-collapse in GaN-based devices due to the 2DEG depletion induced by the negative charge build up occurring in the C-doped GaN buffer when devices are subjected to high operating voltages. The assumption of an acceptor trap located at $0.9 \mathrm{eV}$ from the $\mathrm{GaN}$ VB was also able to reproduce by means of numerical simulations the currentcollapse in GaN Schottky Barrier Diode (SBD) grown on Cdoped $\mathrm{GaN}$ buffer [8]. Drain current transient measurements carried out on ungated $\mathrm{AlGaN} / \mathrm{GaN}$ structures with C-doped $\mathrm{GaN}$ buffer layer have shown the presence of a $0.86 \mathrm{eV}$ thermally activated current decrease when applying moderate negative substrate voltage levels which has been explained by means of hole conduction in the GaN VB [9]. Recently, it was shown experimentally in [10] that device on resistance recovery after off-state high bias conditions in C-doped GaN buffer devices was thermally activated with an $\mathrm{E}_{\mathrm{A}}=0.93 \mathrm{eV}$ and it was suggested that this phenomena might be related to the emission of electrons captured in carbon-related buffer traps during off-state high bias conditions.

Nevertheless, a clear and quantitative correlation between experimental and numerical simulation results concerning trapping phenomena linked to $\mathrm{C}$-doped $\mathrm{GaN}$ buffer layers has not yet been presented. Aim of this paper is thus to study both by experimental measurements and numerical simulations the role of carbon doping in $\mathrm{GaN}$ layers in order to gain insights in the trap levels formed by the incorporation of carbon as well as to provide quantitative results concerning the simulation parameters that have to be adopted in order to take into account carbon related trap levels. This last point is particularly critical in order to use numerical simulations as a tool for the prediction and/or evaluation of GaN-based power HEMTs operation. 


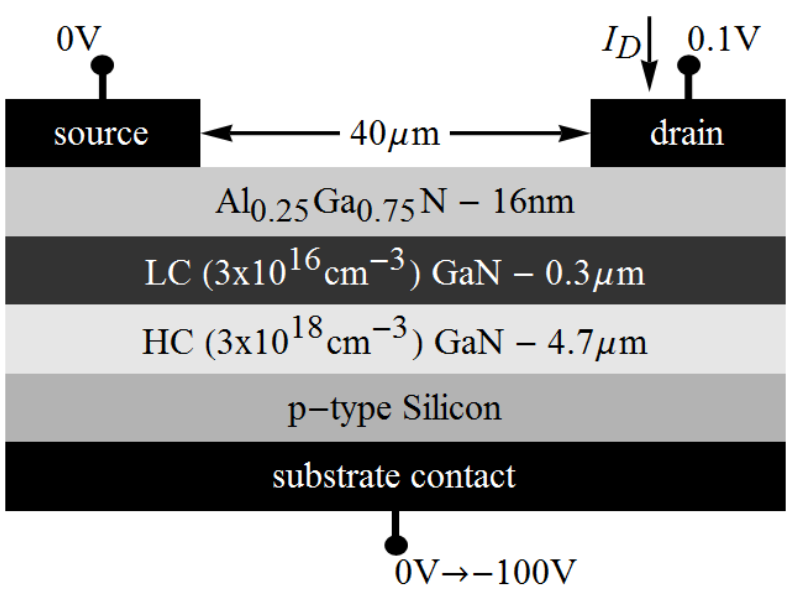

Fig. 1. Cross- section of the tested and simulated ungated $\mathrm{AlGaN} / \mathrm{GaN}$ HEMTs structures. Bias voltage applied during the drain current transient measurement after applying a negative voltage step from $0 \mathrm{~V}$ to $-100 \mathrm{~V}$ to the wafer substrate are also reported.

The paper is thus organized as reported as follows.

Section II will briefly describe the devices used in this work and the experimental measurements carried out. Particularly, the main results obtained is that when applying a negative substrate voltage to an ungated $\mathrm{AlGaN} / \mathrm{GaN}$ structure a thermally activated drain current decrease is observed with $\mathrm{E}_{\mathrm{A}}=0.9 \mathrm{eV}$.

Section III describes the setup of the numerical simulations carried out in order to explain the experimental data. Three different traps configuration scenarios will be considered.

Section IV presents the numerical simulation results obtained by considering the three traps scenarios. Only when an acceptor trap level at $0.9 \mathrm{eV}$ from $\mathrm{GaN}$ valence-band is considered, scenario B, a qualitative agreement between simulated and measured data can be observed

Finally, section $\mathrm{V}$ deals with the quantitative fitting by means of numerical simulations of the experimental data. It will be shown that a nice quantitative fitting can be obtained only if a traps concentration significantly lower than the nominal C-doping level is considered.

\section{EXPERIMENTAL RESULTS}

Experimental measurements and numerical simulations presented were carried out on ungated $\mathrm{AlGaN} / \mathrm{GaN}$ HEMTs structures grown by MOCVD on a $\mathrm{Si}(111)$ p-type substrate with a source/drain spacing of $40 \mu \mathrm{m}$, see figure 1 . The epilayers consisted of a nucleation layer, a $4.7 \mu \mathrm{m}$ thick $\mathrm{GaN}$ buffer with a $3 \times 10^{18} \mathrm{~cm}^{-3}$ carbon doping concentration (HC), a $0.3 \mu \mathrm{m}$ thick GaN layer with a $3 \times 10^{16} \mathrm{~cm}^{-3}$ carbon doping concentration (LC) followed by a $15 \mathrm{~nm} \mathrm{Al}_{0.25} \mathrm{Ga}_{0.75} \mathrm{~N}$ barrier layer. Ohmic contacts were formed by Ti/Al-based metallization defined by means of a lift-off process [11].

Temperature-dependent transient backgating measurements were performed on the ungated structure in order to identify trap levels within the GaN buffer layer $[12,13]$. In fact, the tested structure has the benefit that the occupancy of trap levels located at the $\mathrm{AlGaN} / \mathrm{GaN}$ interface, $\mathrm{AlGaN}$ barrier and device surface will not change thanks to the 2DEG shielding

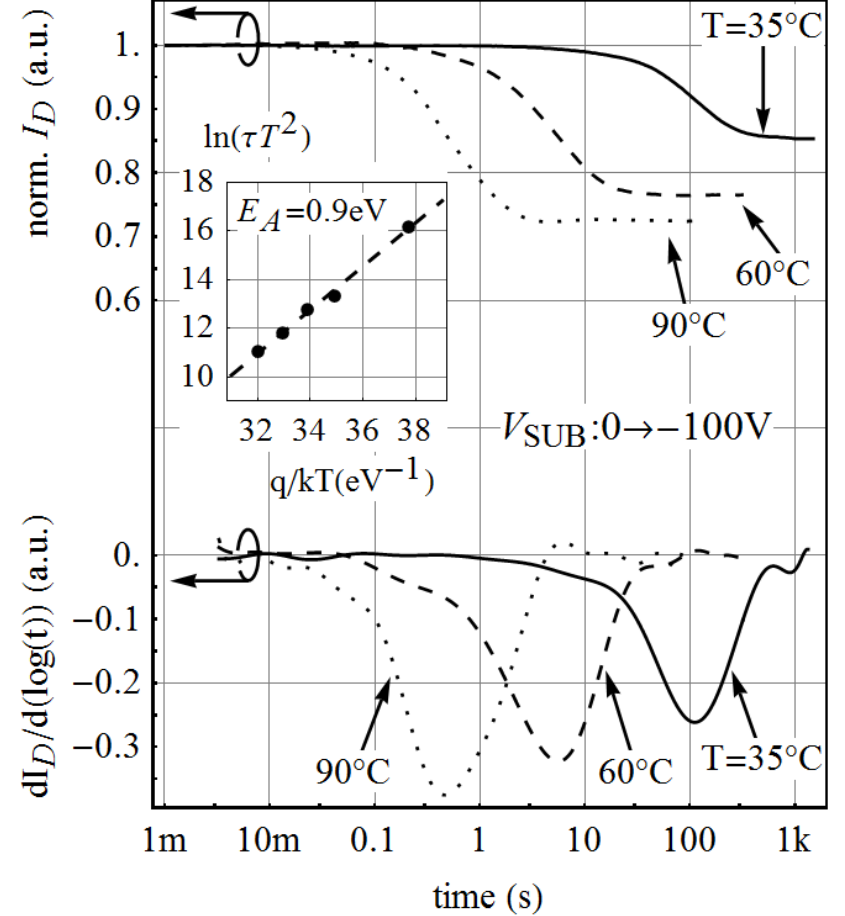

Fig. 2. Recorded normalized drain current transients at $\mathrm{V}_{\mathrm{DS}}=0.1 \mathrm{~V}$ obtained when applying a negative voltage step from $0 \mathrm{~V}$ to $-100 \mathrm{~V}$ to the wafer backside. Increasing the temperature yields a speed-up of the current transient observed suggesting that the observed phenomena might be related to a thermally activated process. Analysis of the $\mathrm{dI} / \mathrm{d}(\log (\mathrm{t}))$ signals showed clear peaks used for the extraction of the time constants $\tau$. The inset reports the Arrhenius plot obtained which yielded an activation energy $E_{A}=0.9 \mathrm{eV}$.

effect as long as the applied source and drain potentials remain constant and the 2DEG is formed, i.e. on-state conditions. As a consequence the tested structure, during current transient backgating measurements, will be sensitive to occupancy variation only of trap levels located within the GaN buffer layers as long as the 2DEG is not fully depleted.

The time-dependent drain current $I_{D}$, recorded after switching the substrate bias $\mathrm{V}_{\mathrm{SUB}}$ from 0 to $-100 \mathrm{~V}$ at the time $\mathrm{t}=0 \mathrm{~s}$, was measured at $\mathrm{V}_{\mathrm{DS}}=0.1 \mathrm{~V}$ (with the source grounded) in order to reduce self-heating effects. Said measurements were performed at temperatures within the $35^{\circ} \mathrm{C}$ to $90^{\circ} \mathrm{C}$ range. Measured $I_{D}$ values were then normalized with respect to the measured $I_{D}$ at $1 \mathrm{~ms}$ from the beginning of the transient.

The normalized transients of $\mathrm{I}_{\mathrm{D}}$ measured at $\mathrm{T}=35,60$ and $90^{\circ} \mathrm{C}$ are depicted in figure 2 . At $\mathrm{T}=35^{\circ} \mathrm{C}, \mathrm{I}_{\mathrm{D}}$ experienced a decrease of approximately $15 \%$ with an associated time constant of $100 \mathrm{~s}$. Increasing the temperature induced a speedup in the current-transient suggesting that the observed phenomena might be related to a thermally activated process. Clear peaks were also observed when analyzing the $\mathrm{dI}_{\mathrm{D}} / \mathrm{d}(\log (\mathrm{t}))$ signals, see figure 2 , related to the transients evaluated at different temperature were then analyzed in order to extract the emission time constants $\tau$ [14] of the thermally activated process causing the observed current decrease. If at this point we suppose that the thermally activated process corresponds to the emission of electrons from a trap level located at $\mathrm{E}_{\mathrm{C}}-\mathrm{E}_{\mathrm{A}}$ (where $\mathrm{E}_{\mathrm{C}}$ is the conduction band energy and $E_{A}$ is the activation energy of the trap), or alternatively the 
emission of holes from a trap level located at $\mathrm{E}_{\mathrm{V}}+\mathrm{E}_{\mathrm{A}}$ (where $E_{V}$ is the valence band energy), the energy $E_{A}$ can be extracted as the slope of the Arrhenius plot of $\ln \left(\tau \mathrm{T}^{2}\right) \mathrm{vs} \mathrm{q} /(\mathrm{kT})$ where $\tau$ represents the time constant measured at the temperature $\mathrm{T}, \mathrm{q}$ is the electron charge and $\mathrm{k}$ is the Boltzmann constant. As can be seen in the inset of figure 2 , the extracted $\mathrm{E}_{\mathrm{A}}$ was $0.9 \mathrm{eV}$.

The $0.9 \mathrm{eV}$ number obtained in this paper well correlates with the $0.86 \mathrm{eV}$ experimental data presented in [9], which has been obtained in similar measurements conditions, i.e. monitoring drain current decrease when applying negative $\mathrm{V}_{\text {SUB }}$ voltages although the $\mathrm{V}_{\text {SUB }}$ in [9] was $-25 \mathrm{~V}$ while in this work it was $-100 \mathrm{~V}$. We might speculate that the mechanism observed in our devices could be similar to that observed in [9] for $\mathrm{V}_{\mathrm{SUB}}=-25 \mathrm{~V}$. Nevertheless, our interpretation of the physical mechanism leading to the observed drain current decrease is different from that proposed in [9]. As it will be demonstrated in the following sections by means of numerical simulations, our proposed explanation relies on a holeemission process from a Carbon-related acceptor-like trap which is different from the hole conduction mechanism proposed in [9].

When comparing our extracted $0.9 \mathrm{eV}$ activation energy with the $0.93 \mathrm{eV}$ experimental data presented in [10], these two numbers cannot be directly correlated since the $0.93 \mathrm{eV}$ has been obtained in different conditions, i.e. by monitoring a $\mathrm{R}_{\mathrm{DSon}}$ recovery, i.e. a drain current increase, by applying $\mathrm{V}_{\mathrm{SUB}}=0 \mathrm{~V}$ after a $100 \mathrm{~s}$ stress condition at $\mathrm{V}_{\mathrm{SUB}}=-100 \mathrm{~V}$. As a consequence the data reported in [10] and that reported in this work cannot be properly compared since the activation energy in [10] has been derived from a completely different measurement method where other physical mechanisms than those occurring in the devices tested in this paper might be present.

Before moving to the next section we would like to briefly comment on the meaning of the activation energy extracted from the Arrhenius plot. The value extracted from the Arrhenius plot itself is not able to distinguish between a hole or an electron trap. The $\mathrm{E}_{\mathrm{A}}$ value extracted is related to the distance of the trap level from the conduction-band edge in case of an electron trap or from the valence-band edge in case of a hole trap. Nevertheless some speculations can be made on the experimental data obtained in order to understand if the trap causing them will be an electron-trap or a hole-trap one. We would like to stress however that in order to prove said speculations numerical simulations needs to be performed as it will be shown in section III and IV. Drain current is decreasing with time, meaning that the number of electrons in the $2 \mathrm{DEG}$ is decreasing as a consequence of the charge variation induced by the trap emission process. As a consequence, only hole emission, i.e. a loss of positive charges in the buffer or equivalently the buildup of a negative charge in the buffer, is consistent with the observed phenomena.

\section{NUMERICAL SIMULATIONS}

In order to gain insights in the physical mechanisms leading to the observed current decrease after applying a negative substrate voltage step, numerical simulations were carried out with the commercial software DESSIS-ISE. For all the simulations, an n-type GaN background doping with a $10^{16} \mathrm{~cm}^{-3}$ concentration was adopted, while three different traps configuration scenarios were considered for the LC and HC GaN layers, see figure 3. Said scenarios will now be described in detail in the following subsections.

\section{A. Traps scenario $A$}

Scenario A refers to one of the possible compensation mechanism induced by Carbon doping which predicts the auto-compensation through interplay of $\mathrm{C}_{\mathrm{N}}-\mathrm{C}_{\mathrm{Ga}}$ states incorporated during growth with comparable concentration $[3,15]$. According to this model, the Fermi level is predicted to be pinned at around mid-gap thus making the C-doped buffer to behave as an almost ideal semi-insulating (SI) layer.

For simulating the effects of this traps configuration, a donor-like trap was introduced at $\mathrm{E}_{\mathrm{CGa}}=0.11 \mathrm{eV}$ from the $\mathrm{CB}$ together with an acceptor-like trap $\mathrm{E}_{\mathrm{CN}}$ at $3.28 \mathrm{eV}$ from the GaN CB. Trap densities were set according to the carbon doping levels simply by equally splitting them within the two levels, i.e. for the $3 \times 10^{18} \mathrm{~cm}^{-3} \mathrm{HC}$ layer, $1.5 \times 10^{18} \mathrm{~cm}^{-3}$ traps were assigned to the level at $\mathrm{E}_{\mathrm{CGa}}$ and the remaining $1.5 \times 10^{18} \mathrm{~cm}^{-3}$ traps were assigned to the level at $\mathrm{E}_{\mathrm{CN}}$.

\section{B. Traps scenario $B$}

Traps scenario $\mathrm{B}$ refers to the compensating mechanisms induced by dominant $\mathrm{C}_{\mathrm{N}}$ acceptor states [16-18]. Particularly, as reported in [18], using hybrid density functional calculations it has been shown that carbon on the nitrogen site is in fact a deep acceptor. Calculations reported in [18,19] also yielded a (0/-) charge-state transition level $\mathrm{E}=0.9-1.1 \mathrm{eV}$ above the VB. According to this model the Fermi level will result to be pulled in the bottom half of the band-gap turning the GaN buffer into a weakly p-type region [20].

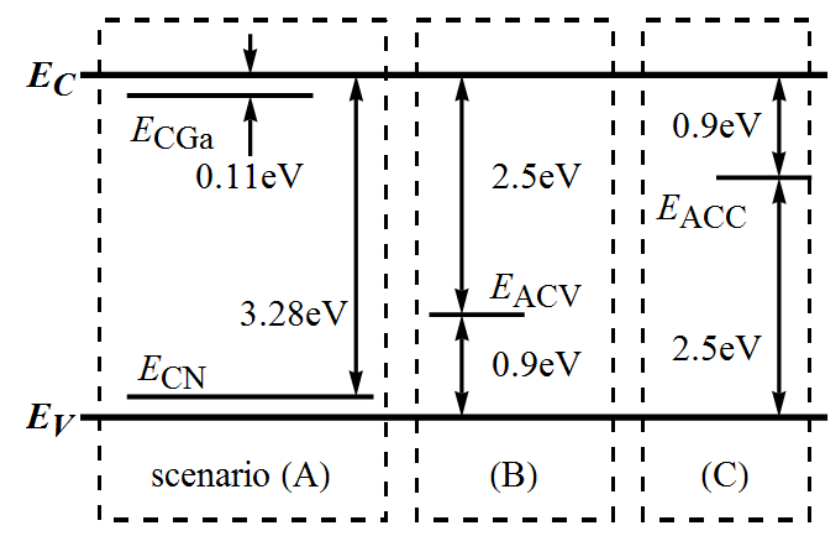

Fig. 3. Three possible traps scenario were considered for reproducing the experimental results by means of numerical simulations. Scenario A and B refer to two of the possible compensation mechanisms induced by Carbon doping in GaN layers. Particularly, scenario (A) represents the autocompensation through interplay of $\mathrm{C}_{\mathrm{N}}-\mathrm{C}_{\mathrm{Ga}}$ states while scenario (B) represents the compensation through a dominant $\mathrm{C}_{\mathrm{N}}$ acceptor state. On the other hand, scenario (C) is not related in principle to known Carbon-doping related compensation mechanisms but it has been considered due to the experimentally extracted $0.9 \mathrm{eV}$ activation energy of the thermally activated drain current transient decrease. 
Simulations adopting this model were carried out by introducing an acceptor-like trap level at $\mathrm{E}_{\mathrm{ACV}}=0.9 \mathrm{eV}$ from the GaN VB, with trap densities set according to the carbon doping levels of the structure.

\section{Traps scenario $C$}

Traps scenario C, differently from scenario A and B, has not been chosen according to expected trapping levels related to Carbon doping within $\mathrm{GaN}$ layers. Based on the $0.9 \mathrm{eV}$ activation energy experimentally extracted an acceptor-like trap level was considered at $0.9 \mathrm{eV}$ from the $\mathrm{GaN} \mathrm{CB}$.

Simulations adopting this model were carried out by introducing an acceptor-like trap level at $\mathrm{E}_{\mathrm{ACC}}=0.9 \mathrm{eV}$ from $\mathrm{CB}$, with trap densities set according to the structure carbon doping levels.

\section{Simulated Current Transients}

Simulated current transient according to the three traps scenario described in the previous section and the experimental results obtained at $\mathrm{T}=35^{\circ} \mathrm{C}$ are depicted in figure 4. The results obtained for each of the three traps scenarios considered will now be discussed as follows.

\section{A. Scenario A simulation results}

Scenario A, figure 4 dotted line, did not exhibit any variation in the drain current versus time. This is expected since the two $\mathrm{E}_{\mathrm{CGa}}$ and $\mathrm{E}_{\mathrm{CN}}$ levels are fully-ionized regardless of the applied substrate voltage. As a consequence the negative charge build-up in the buffer, which is required in order to induce the observed drain current decrease, will never come into place.

\section{B. Scenario B simulation results}

Scenario B, see figure 4 dot-dashed line, yielded a significant decrease in the simulated current level, actually turning off completely the 2DEG. Again, the trends is expected since $\mathrm{E}_{\mathrm{ACV}}$ will behave as an hole trap and it will be briefly discussed. When a negative $\mathrm{V}_{\mathrm{SUB}}$ is applied the depletion region within the GaN buffer layers will increase its extension. Trapped holes within said depletion region should then be swept away, although this mechanism will have to follow the timing related to the emission process of the hole traps. As time passes by, holes are emitted, leaving negatively ionized acceptors within the depleted region. A negative charge build-up will thus occur in the buffer inducing a decrease in the 2DEG density and consequently in the current level with time constant related to the hole emission process.

\section{Scenario $C$ simulation results}

Scenario C, see figure 4 dashed line, resulted in a complete opposite trend with respect to scenario $\mathrm{B}$ and experimental results, yielding an increase of the current during the simulated transient. The charge dynamic involved during the current increase can be briefly explained as follows. When $\mathrm{V}_{\mathrm{SUB}}=0 \mathrm{~V}$ traps are filled by electron in the buffer layer in order to compensate the effect of the n-type background doping resulting in the Fermi level being pinned at $0.9 \mathrm{eV}$ from the $\mathrm{CB}$. As $\mathrm{V}_{\mathrm{SUB}}$ is pulled to negative values the depletion

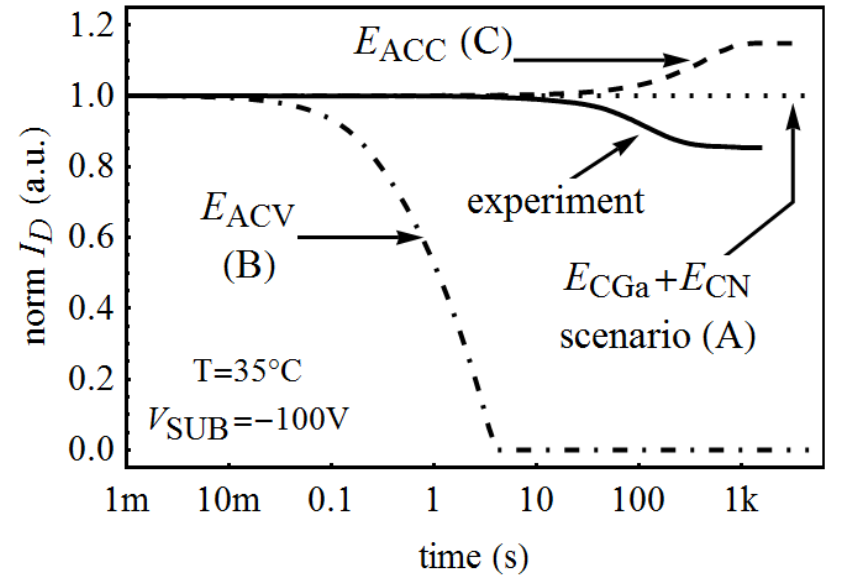

Fig. 4. Comparison amongst experimental and simulated normalized drain current transients at $\mathrm{T}=35^{\circ} \mathrm{C}$ when applying a negative voltage step from $0 \mathrm{~V}$ to $-100 \mathrm{~V}$ to the substrate: experimental results (solid line); simulated considering scenario A (dotted line); simulated considering scenario B (dashdotted line) and simulated considering scenario $\mathrm{C}$ (dashed line). Amongst the three scenario considered only scenario $\mathrm{B}$, corresponding to a compensation mechanism through a dominant $\mathrm{C}_{\mathrm{N}}$ acceptor state at $0.9 \mathrm{eV}$ from the $\mathrm{GaN}$ $\mathrm{VB}$, yielded a decrease in the drain current level.

region forming within the buffer layer sweeps away the trapped electrons, with time constants related to their emission process, inducing a positive charge build up in the buffer due to the presence of the background donors which are no longer neutralized by the trapped electrons.

We can thus conclude this preliminary qualitative analysis by stating that amongst the three model considered only scenario $\mathrm{B}$ is able to predict the measured current decrease versus time. Hole traps associated with the $C_{N}$ acceptor states thus seem to be the main responsible of the experimentally observed thermally activated drain current decrease when negative substrate voltage levels are applied to the tested structures.

\section{Quantitative FitTing of ExPERIMENTAL Data}

As observed in the previous section, amongst the three different traps scenarios considered only scenario B was able to reproduce a drain current decrease versus time.

While this result qualitatively follows the experimental one, simulations are predicting a larger current variation if compared to the measured one. This quantitative discrepancy between measured ad simulated drain currents suggests that traps concentrations used in simulation might be larger than those actually involved in the real tested device.

Since we observed that scenario A yielded no current variation while only scenario $\mathrm{B}$ was yielding a decrease in current level, additional numerical simulation were carried out by adjusting the traps concentration within the simulated structure according to the following method. Naming $x$ a certain fraction of the nominal Carbon doping levels, i.e. $3 \times 10^{16} \mathrm{~cm}^{-3}$ for the LC and $3 \times 10^{18} \mathrm{~cm}^{-3}$ for the HC layers respectively, the number of trap level related to scenario $\mathrm{B}$ was set equal to $x$, while the remaining $1-x$ were equally distributed within the shallow-donor and shallow-acceptor trap 
levels of scenario A.

As can be seen in figure 5, varying $x$ allowed us to quantitatively reproduce the experimentally recorded transient which was adequately fitted for an $x$ value equal to $0.6 \%$, i.e. corresponding to a $1.8 \times 10^{16} \mathrm{~cm}^{-3} \mathrm{E}_{\mathrm{ACV}}$ trap concentration in the $\mathrm{HC}$ buffer layer. The capture cross-section used in the simulations for the $\mathrm{E}_{\mathrm{ACV}}$ trap level was $1.3 \times 10^{-14} \mathrm{~cm}^{-2}$. Said capture cross-section value was chosen in order to match the experimental current transients and to obtain a nice overlap between the experimental, see inset of figure 2, and simulated, inset of figure 5, Arrhenius plots. Lower values of the capture cross-section leaded to slower transients and vice versa, with $1.3 \times 10^{-14} \mathrm{~cm}^{-2}$ being the capture cross-section value which yielded the nice fit with the experimental data reported in figure 5. Simulations with $x=0.6 \%$ were also carried out at different temperature, and, by adopting the method based on the $\mathrm{dI}_{\mathrm{D}} / \mathrm{d}(\log (\mathrm{t}))$ signals peaks extraction, the Arrhenius plot reported in the inset of figure 5 was calculated, yielding a $0.9 \mathrm{eV}$ activation energy.

Some comments are now needed in order to discuss the large discrepancy, approximately 2 orders of magnitude, between the nominal carbon doping values and those required in order to reproduce the current transients with numerical simulation. A similar difference between the nominal carbon doping and the effective trap density has also been observed in [17] when studying the deep levels in n-GaN Carbon doped layer by means of Minority Carrier Transient Spectroscopy (MCTS) measurements. Particularly, the signature of a holetrap at $0.86 \mathrm{eV}$ from VB was reported in [17], which is in agreement with our findings, both on low-carbon (2 to $\left.5 \times 10^{16} \mathrm{~cm}^{-3}\right)$ and high-carbon $\left(1 \times 10^{17} \mathrm{~cm}^{-3}\right)$ doped n-type GaN layers. The extracted trap densities in [17] were also $1.8 \times 10^{14} \mathrm{~cm}^{-3}$ and $2.2 \times 10^{15} \mathrm{~cm}^{-3}$ for the low-carbon and highcarbon samples, respectively. The increase in trap density with increasing carbon doping clearly correlated the hole-trap with the carbon doping in the experiment carried out in [17]. Moreover, similar to our findings, they also observed approximately a 2 orders of magnitude ratio between the effective carbon doping and the hole-trap concentration. In agreement with our results, Photo Capacitance and DLTS measurements reported in [21] also demonstrated that less than $2 \%$ of the nominal carbon concentration created traps at a $0.9 \mathrm{eV}$ level from the $\mathrm{GaN}$ valence band. Concerning the remaining part of the Carbon doping, it was reported in [21] that $15-20 \%$ is related to different levels and the other part, about $80 \%$, is still not allocated.

This result further supports our findings based on the quantitative fitting of the experimental data where only $0.6 \%$ of the Carbon doping participates to the depletion of the 2DEG.

\section{CONCLUSIONS}

To the best of the authors knowledge we have provided, for the first time, a clear and quantitative explanation of drain current decrease transients induced by Carbon related buffer traps which only involves the hole-emission physical process related to an acceptor trap located at $0.9 \mathrm{eV}$ from the $\mathrm{GaN} \mathrm{VB}$,

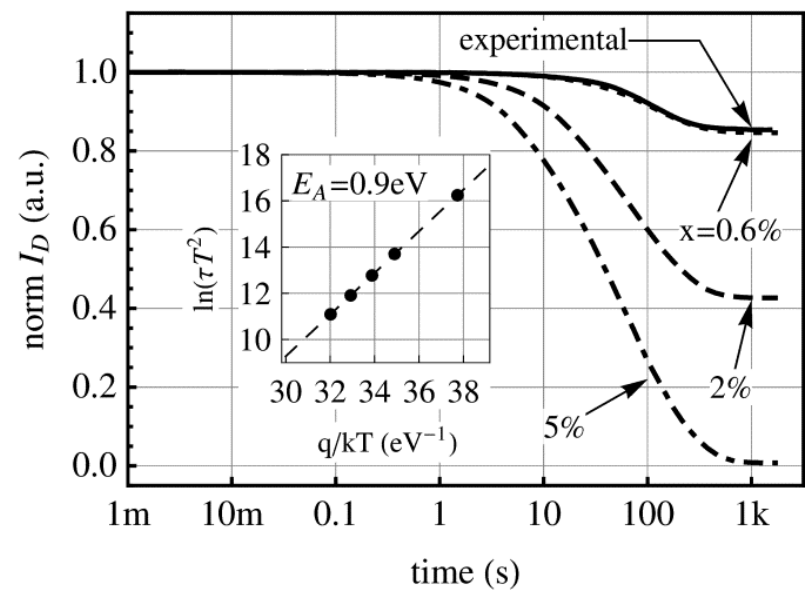

Fig. 5. Simulated normalized drain current transients obtained by varying the fraction $x$ of Carbon doping associated to the scenario B trap level density. A nice fitting of the experimental data, solid line, is obtained by means of numerical simulations when $x=0.6 \%$, dotted line. The inset reports the calculated Arrhenius plot obtained by carrying out simulated transients at different temperatures yielding a $0.9 \mathrm{eV}$ activation energy.

which is one of the known levels forming within C-doped $\mathrm{GaN}$ layers [16-18].

The current reduction in the tested ungated $\mathrm{AlGaN} / \mathrm{GaN}$ structures during back-gating transient measurements is related to a hole-emission process from an acceptor-like holetrap level with a density corresponding to $0.6 \%$ of the nominal Carbon doping concentration.

Both the trap energy as well as the ratio between the trap density and the nominal Carbon doping are also in agreement with previous findings obtained by means of different measurements techniques, with respect to that adopted in this work, on C-doped GaN layers [17,21].

The results proposed in this paper can potentially provide insights in the physical mechanisms involved also in the performance degradation of GaN-based HEMTs grown on Cdoped buffers. The measurement conditions used here on ungated structures will correspond, in first approximation, to those experienced by HEMT devices in their gate-drain access region when large positive drain voltage are applied in offstate conditions with their substrate grounded. As a consequence, the traps configuration scenario which fitted the experimental results could be used to predict and/or investigate the performance degradation in GaN-based HEMTs with C-doped GaN buffer layers.

\section{REFERENCES}

[1] R. Mitova, R. Ghosh, U. Mhaskar, D. Klikic, M. X. Wang and A. Dentella, "Investigations of 600-V GaN HEMT and GaN Diode for Power Converter Applications," IEEE Trans. On Power Electronics, vol. 29, no. 5, pp. 2441-2452, May 2014

[2] Y.-F. Wu, J. Gritters, L. Shen, R. P. Smith and B. Swenson, "kV-Class GaN-on-Si HEMTs Enabling 99\% Efficiency Converter at $800 \mathrm{~V}$ and 100 kHz," IEEE Trans. On Power Electronics, vol. 29, no. 6, pp. 26342637, Jun. 2014.

[3] A. Armstrong, C. Poblenz, D. S. Green, U. K. Mishra, J. S. Speck and S. A. Ringel, "Impact of substrate temperature on the incorporation of carbon-related defects and mechanism for semi-insulating behavior in GaN grown by molecular beam epitaxy," Appl. Phys. Lett., vol. 88, no. 8, pp. 082111-4, Feb. 2006.

[4] E. Bahat-Treidel, F. Brunner, O. Hilt, E. Cho, J. Wurfl and G. Trankle, "AlGaN/GaN/GaN:C Back-Barrier HFETs With Breakdown Voltage of 
Over $1 \mathrm{kV}$ and Low $\mathrm{R}_{\mathrm{ON}} \times \mathrm{A}, "$ IEEE Trans. On Electron Devices, vol. 57, no. 11 , pp. $3050-3058$, Nov. 2010.

[5] J. Würfl, O. Hilt, E. Bahat-Treidel, R. Zhytnytska, P. Kotara, F. Brunner, $\mathrm{O}$. Krueger and $\mathrm{M}$. Weyers, "Techniques towards GaN power transistors with improved high voltage dynamic switching properties," IEEE International Electron Device Meeting (IEDM), pp. 144-147, 2013.

[6] P. Moens, P. Vanmeerbeek, A. Banerjee, J. Guo, C. Liu, P. Coppens, A. Salih, M. Tack, M. Caesar, M. J. Uren, M. Kuball, M. Meneghini, G Meneghesso and E. Zanoni, "On the Impact of Carbon-Doping on the Dynamic Ron and Off-state Leakage Current of 650V GaN Power Devices," IEEE 27th International Symposium on Power Semiconductor Devices and ICs (ISPSD), pp. 37-40, 2015.

[7] M. J. Uren, J. Möreke, M. Kuball, "Buffer Design to Minimize Current Collapse in GaN/AlGaN HFETs," IEEE Trans. On Electron Devices, vol. 59, no. 12, pp. 3327-3333, Dec. 2012.

[8] J.A. Croon, G. A. M. Hurkx, J. J. T. M. Donkers and J. Šonský, "Impact of the backside potential on the current collapse of GaN SBDs and HEMTs," IEEE 27th International Symposium on Power Semiconductor Devices and ICs (ISPSD), pp. 365-368, 2015

[9] M. J. Uren, M. Cäsar, M. A. Gajda, and M. Kuball, " Buffer transport mechanisms in intentionally carbon doped $\mathrm{GaN}$ heterojunction field effect transistors," Appl. Phys. Lett., vol. 104, no. 26, pp. 263505-1-4, Jun. 2014.

[10] G. Meneghesso, M. Meneghini, R. Silvestri, P. Vanmeerbeek, P. Moens and Enrico Zanoni, "High voltage trapping effects in GaN-based metalinsulator-semiconductor transistors," Jap. J. Appl. Phys., vol. 55, no. 1S, pp. 01AD04-1-5, Jan. 2016.

[11] F. Iucolano, G. Greco, F. Roccaforte, "Correlation between microstructure and temperature dependent electrical behavior of annealed $\mathrm{Ti} / \mathrm{Al} / \mathrm{Ni} / \mathrm{Au}$ Ohmic contacts to $\mathrm{AlGaN} / \mathrm{GaN}$ heterostructures," Appl. Phys. Lett., vol. 103, no. 20, pp. 201604-1-4, Nov. 2013.

[12] M. Marso, M. Wolter, P. Javorka, P. Kordoš and H. Lüth, "Investigation of buffer traps in an $\mathrm{AlGaN} / \mathrm{GaN} / \mathrm{Si}$ high electron mobility transistor by backgating current deep level transient spectroscopy," Appl. Phys. Lett., vol. 82, no. 4, pp. 633-635, Jan. 2003

[13] C. Zhou, Q. Jiang, S. Huang and K. J. Chen, "Vertical Leakage/Breakdown Mechanisms in AlGaN/GaN-on-Si Devices," IEEE Electron Device Letters, vol. 33, no. 8, pp. 1132-1134, Aug. 2012.

[14] M. Tapajna, R. J. T. Simms, Y. Pei, U. K. Mishra and M. Kuball, "Integrated Optical and Electrical Analysis: Identifying Location and Properties of Traps in AlGaN/GaN HEMTs During Electrical Stress," IEEE Electron Device Letters, vol. 31, no. 7, pp. 662-664, Jul. 2010.

[15] A. F. Wright, "Substitutional and interstitial carbon in wurtzite GaN," J. Appl. Phys., vol. 92, no. 5, pp. 2575-2585, Sept. 2002.

[16] A. Armstrong, A. R. Arehart, D. Green, U. K. Mishra, J. S. Speck and S. A. Ringel, "Impact of deep levels on the electrical conductivity and luminescence of gallium nitride codoped with carbon and silicon," $J$. Appl. Phys., vol. 98, no. 5, pp. 053704-1-11, Sept. 2005.

[17] U. Honda, Y. Yamada, Y. Tokuda and K. Shiojima, "Deep levels in nGaN Doped with Carbon Studied by Deep Level and Minority Carrier Transient Spectroscopies," Jap. J. Appl. Phys., vol. 51, no. 4S, pp. 04DF04-1-4, Apr. 2012.

[18] J. L. Lyons, A. Janotti, and C. G. Van de Walle, "Carbon impurities and the yellow luminescence in GaN," Appl. Phys. Lett., vol. 97, no. 15, pp. 152108-1-3, Oct. 2010.

[19] D. O. Demchenko, I. C. Diallo, and M. A. Reshchikov, "Yellow Luminescence of Gallium Nitride Generated by Carbon Defect Complexes," Phys. Rev. Lett., vol. 110, no. 8, 087404-1-5, Feb. 2013.

[20] M. J. Uren, M. Silvestri, M. Cäsar, G. A. M. Hurkx, J. A. Croon, J. Šonský and M. Kuball, "Intentionally Carbon-Doped AlGaN/GaN HEMTs: Necessity for Vertical Leakage Paths," IEEE Electron Device Letters, vol. 35, no. 3, pp. 327-329, Mar. 2014.

[21] T. Tanaka, K. Shiojima, Y. Otoki and Y. Tokuda, "A study on multiple defect states in low-carbon doped $\mathrm{GaN}$ layers and its correlation with AlGaN/GaN high electron mobility transistor operation," Thin Solid Films, vol. 557, pp. 207-211, Apr. 2014. 